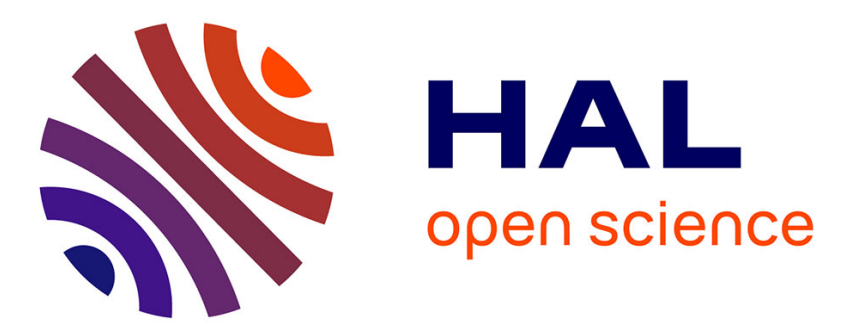

\title{
A new approach to the subcritical cracking of ceramic fibers
}

\author{
Pierre Ladevèze, Martin Genet
}

\section{To cite this version:}

Pierre Ladevèze, Martin Genet. A new approach to the subcritical cracking of ceramic fibers. Composites Science and Technology, 2010, 70 (11), pp.1575. 10.1016/j.compscitech.2010.04.013 . hal00666482

\section{HAL Id: hal-00666482 \\ https://hal.science/hal-00666482}

Submitted on 5 Feb 2012

HAL is a multi-disciplinary open access archive for the deposit and dissemination of scientific research documents, whether they are published or not. The documents may come from teaching and research institutions in France or abroad, or from public or private research centers.
L'archive ouverte pluridisciplinaire HAL, est destinée au dépôt et à la diffusion de documents scientifiques de niveau recherche, publiés ou non, émanant des établissements d'enseignement et de recherche français ou étrangers, des laboratoires publics ou privés. 


\section{Accepted Manuscript}

A new approach to the subcritical cracking of ceramic fibers

Pierre Ladevèze, Martin Genet

PII:

S0266-3538(10)00158-2

DOI:

10.1016/j.compscitech.2010.04.013

Reference:

CSTE 4689

To appear in:

Composites Science and Technology

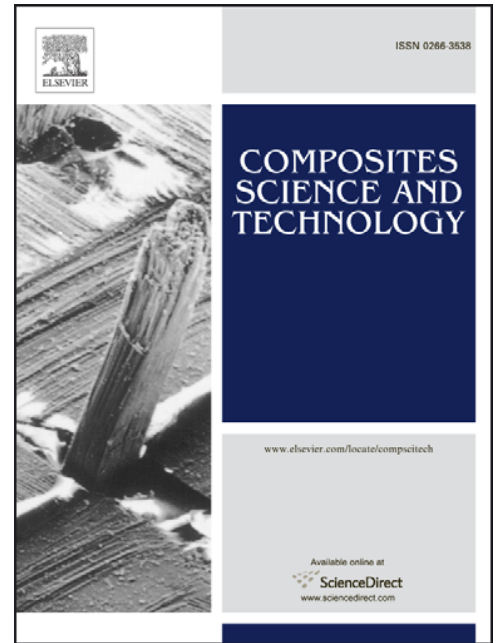

Received Date:

20 August 2009

Revised Date:

19 January 2010

Accepted Date:

17 April 2010

Please cite this article as: Ladevèze, P., Genet, M., A new approach to the subcritical cracking of ceramic fibers, Composites Science and Technology (2010), doi: 10.1016/j.compscitech.2010.04.013

This is a PDF file of an unedited manuscript that has been accepted for publication. As a service to our customers we are providing this early version of the manuscript. The manuscript will undergo copyediting, typesetting, and review of the resulting proof before it is published in its final form. Please note that during the production process errors may be discovered which could affect the content, and all legal disclaimers that apply to the journal pertain. 


\title{
A new approach to the subcritical cracking of ceramic
}

\section{fibers}

\author{
Pierre Ladevèze ${ }^{*, a, 1}$, Martin Genet ${ }^{\mathrm{a}}$ \\ ${ }^{a}$ LMT-Cachan (ENS-Cachan / Paris 6 University / CNRS / UniverSud Paris PRES), 61 \\ avenue du Président Wilson, 94235 Cachan Cedex, France
}

\begin{abstract}
A new modeling approach to subcritical crack propagation (i.e. static fatigue) in ceramics is introduced and applied to the case of ceramic fibers. This approach relies on classical fracture mechanics, in which the zone near the crack's tip is modified by the environment (particularly through oxidation). Chemical reactions and diffusion are introduced naturally, which makes the model highly dependent on the environmental conditions, such as partial oxygen pressure. In some cases, the result comes down to the widely-used Paris-like subcritical crack propagation law. For the general case, a two-parameter model calibrated and validated up to $1,000^{\circ} \mathrm{C}$ for Hi-Nicalon fibers is presented.
\end{abstract}

Key words:

Subcritical cracking, A. Fibers, A. Ceramics, D. Life prediction, E. Modeling

\section{Introduction}

Woven Ceramic Matrix Composites (CMCs) reinforced with long fibers are potential candidates for high-temperature thermal/structural applications [Evans, 1997; Bunsell and Berger, 1999; Dambrine, 2006]. Their structural performance is controlled mainly by the fibers [Lamon, 2001]. Indeed, the fibers within the composite are protected from the surrounding cracks thanks to the mechanical fuse concept [Aveston et al., 1971; Rebillat et al., 1998]. However, once the surrounding matrix has cracked, the fibers are no longer protected from the aggressive environment: air (actually, oxygen, water, etc.) can penetrate the crack network and damage the fibers through oxidation or corrosion [Evans et al., 1996; Forio et al., 2004]. There have been attempts to introduce an environmental protection of the fibers within the composite (using, e.g., self-healing multilayered ceramic matrices or fiber treatments, referred to

\footnotetext{
${ }^{*}$ Corresponding author

${ }^{1}$ EADS Foundation Chair "Advanced Computational Structural Mechanics"
} 
as chemical fuses), but even then the environmental protection is not complete and the fibers remain one of the key factors in the material's lifetime [Cluzel et al., 2008; Gauthier and Lamon, 2009].

The study of fibers lifetime under given environmental conditions is denoted static fatigue analysis because most works use the Paris law as in classical fatigue analysis, or subcritical cracking. Subcritical propagation, also known as slow crack growth, has been widely studied in the literature, especially for ceramics [Lawn, 1974; Wiederhorn et al., 1980; Wachtman, 1996; Evans et al., 1996; Hild et al., 1996; Gauthier and Lamon, 2009]. These works describe the crack's propagation using a Paris-like law relating the crack's velocity to the crack's stress intensity factor calculated under the assumption of elastic behavior. This law, which depends on the temperature, agrees fairly well with experimental results [Wiederhorn et al., 1980; Wachtman, 1996], but cannot be applied directly to varying environmental conditions such as oxygen or water concentration around the fibers. Moreover, it was found [Lawn, 1974; Wachtman, 1996; Evans et al., 1996; Laforêt and Lamon, 2008] that as the temperature increases two different propagation stages take place. In the first stage, called the reaction-controlled stage, the crack's tip is damaged directly. The second stage, called the diffusion-controlled stage, is different in the sense that oxygen and water, prior to damaging the crack's tip, must first diffuse through an oxide layer formed on the surface of the fiber. Corresponding models can be found in [Lawn, 1974] for the fibers and in [Laforêt and Lamon, 2008] for the multifilament tow.

The alternative approach to subcritical propagation introduced here relies on classical fracture mechanics, in which the environment modifies the damage zone only at the crack's tip, then drives the progressive propagation of the crack. Pure elastic behavior without creep or mechanical damage is assumed, which is what is observed within the restricted scope of this study, as will be seen further on. The proposed model for subcritical propagation does not depend explicitly on temperature, but depends only on the total oxygen flux reacting at the crack's tip (which, in turn, depends on the temperature, the oxygen pressure, etc.). Consequently, the model is valid for any temperature or oxygen pressure, which can be functions of time, and the only difficulty consists in modeling the oxidizing environment of the crack properly. Only two parameters are needed in order to use the model under varying environmental conditions.

If the crack's growth is relatively slow, one can achieve a very good approximation; in the case of constant environmental conditions, this leads to the widely-used Paris-like law for the lifetime. For Hi-Nicalon fibers up to $1,000^{\circ} \mathrm{C}$ in dry environment, we introduced the chemical phenomena observed (reaction and diffusion) through simple models in order to define the true oxygen flux reacting at the crack's tip. The resulting calibrated model was validated using the experimental data reported in [Gauthier and Lamon, 2009]. 


\section{The new approach}

\subsection{Defect modeling}

In this paper, classical Weibull theory and associated idealizations are used to describe the distribution of defects over the fiber's surface [Lamon, 2007; Gauthier and Lamon, 2009].

In the Weibull theory, the strength of a fiber of length $L$, denoted $\sigma_{R}$, follows the cumulative density function:

$$
p\left(\sigma_{R}<\sigma\right)=1-\exp \left(-\frac{L}{L_{0}}\left(\frac{\sigma}{\sigma_{0}}\right)^{m_{\sigma}}\right)
$$

where $L_{0}, \sigma_{0}$ and $m_{\sigma}$ are classical Weibull coefficients.

Moreover, the growth of surface defects is generally assumed to be a simple one-dimensional problem in which the size of a defect is described by a scalar parameter (Figure 1). This assumption means that the Stress Intensity Factor (SIF), denoted $K$, is approximately uniform along the crack's tip and that the impact of the environmental conditions on the elasticity coefficients is limited. This fact was proven for Hi-Nicalon fibers up to $1,000^{\circ} \mathrm{C}$ in [Bunsell and Berger, 2000; Song et al., 2000]. Thus, the SIF induced by a surface defect of size $a$ in a fiber subjected to a stress $\sigma$ can be calculated using classical elasticity theory:

$$
K=\sigma Y \sqrt{a}
$$

where $Y=2 / \sqrt{\pi}$ is the shape coefficient associated with the crack assumed to be constant throughout the propagation.

This modeling framework introduces two important quantities (see Figure 1):

- The largest surface defect (whose initial size is denoted $a_{0}$ ) is responsible for fiber failure in statics as well as in static fatigue. During static loading, the SIF reaches the Critical Stress Intensity Factor (CSIF), denoted $K_{c}$, at the largest surface defect when the applied stress reaches the fiber's strength, denoted $\sigma_{R}$. Therefore, the initial size of the largest surface defect is obtained, for any given strength, by:

$$
\sigma_{R} Y \sqrt{a_{0}}=K_{c} \quad \Longrightarrow \quad a_{0}=\left(\frac{K_{c}}{\sigma_{R} Y}\right)^{2}
$$

- Similarly, if the fiber is stressed at $\sigma<\sigma_{R}$, there exists a critical defect size $a_{c}>a_{0}$ for which the SIF reaches the CSIF:

$$
\sigma Y \sqrt{a_{c}}=K_{c} \quad \Longrightarrow \quad a_{c}=\left(\frac{K_{c}}{\sigma Y}\right)^{2}
$$




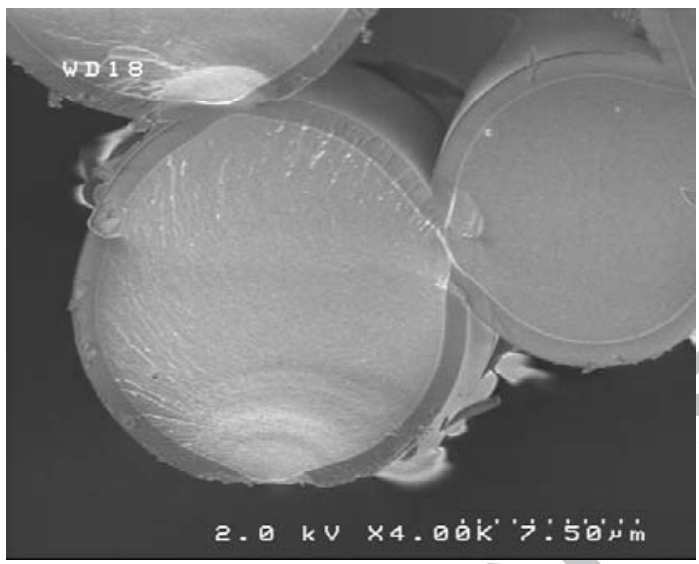

(a) Actual defect, laforet.2008

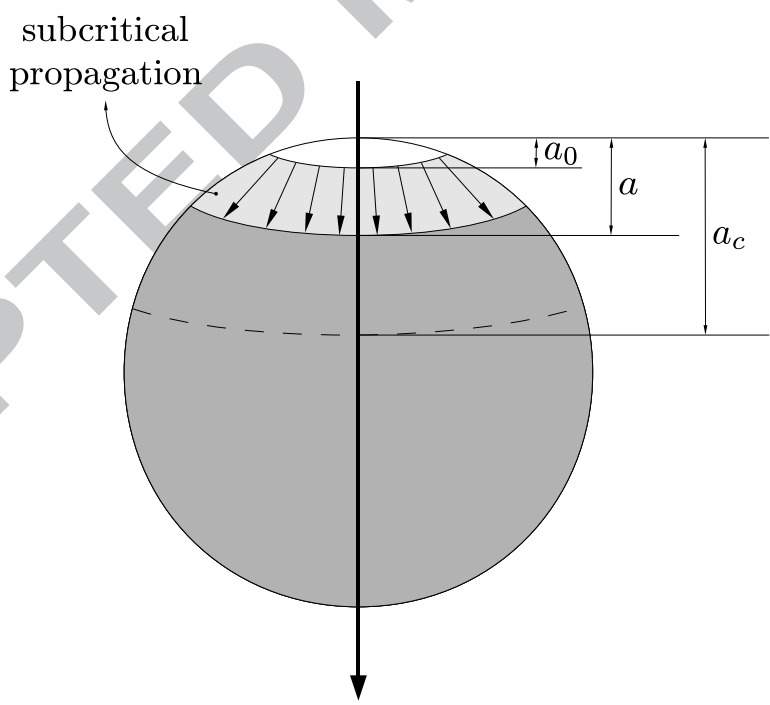

(b) Idealized defect: initial size and critical size

Figure 1: Surface defect in a cross section of a fiber 


\subsection{Subcritical propagation: the classical model}

There have been several attempts at modeling subcritical crack propagation, i.e. deriving a set of equations defining the evolution of the crack's size $a(t)$ from $a_{0}$ to $a_{c}$. The approaches found in the literature [Wiederhorn et al., 1980; Wachtman, 1996; Gauthier and Lamon, 2009] are based on a Paris-like law for the crack's velocity:

$$
\frac{d a}{d t}=v_{0} \cdot K^{n}
$$

where $v_{0}$ and $n$ are parameters which must be calibrated from experimental data. It is worth observing that it is the use of this Paris law which is responsible for the term static fatigue being commonly used in the literature to refer to the subcritical propagation problem.

However, this law has several limitations. First, its physical meaning is not clear: the crack's propagation is only described, but not explained, and it does not follow the principles of fracture mechanics. Second, the temperature dependence induced by chemical reactions is generally handled through a macroscopic exponential law (or Arrhenius law) for $v_{0}$, which requires the introduction of an additional parameter which must be calibrated. Finally, the introduction of external environmental conditions, such as the oxygen concentration, and their variations is not straightforward.

\subsection{Subcritical propagation: the new model}

\subsubsection{Fundamentals}

Propagation law. The basic idea is very simple. The propagation of defects follows classical fracture mechanics:

$$
\left\{\begin{array}{l}
K \leq K_{s c}(a(t), t) \\
\dot{a}(t) \geq 0 \\
\dot{a}(t) \cdot\left(K_{s c}(a(t), t)-K\right)=0
\end{array}\right.
$$

where $K_{s c}$ is the SubCritical Stress Intensity Factor (SCSIF), which is smaller than the CSIF $K_{c}$ because of the oxidation. Let us emphasize that $K_{s c}$, which is a function of time, is defined everywhere within the fiber. However, for the sake of simplicity, we will show that $K_{s c}$ can be assumed to be a function of only one unidirectional space variable (the same variable which describes the crack's size) and time.

The damage law. Now let us consider the dependence of $K_{s c}$ on the environment. The progression of the oxidation reaction which is responsible for the material's damage is described by the reaction rate $\phi_{r}$, which is the most suitable parameter to represent the oxidationinduced degradation of the local mechanical properties (Young's modulus, strength, etc.). For the example of the degradation of the CSIF into the SCSIF, one writes:

$$
\dot{K}_{s c}(x, t)=-f\left(K_{s c}(x, t), T(x, t), \phi_{r}(x, t)\right)
$$


where $f$ is a simple function to be calibrated. It was proven in [Bunsell and Berger, 2000; Song et al., 2000] that for Hi-Nicalon fibers up to $1,000^{\circ} \mathrm{C}$ the direct dependence of the critical properties on the temperature is limited; therefore, it will be disregarded in the following discussion. Then, taking into account the initial conditions:

$$
K_{s c}(x, t=0)=K_{c}
$$

$K_{s c}$ can be written as follows:

$$
K_{s c}(x, t)=K_{c}(1-g(r(x, t))) \quad \text { with } \quad r(x, t)=\int_{0}^{t} \phi_{r}^{m}(x, \tau) d \tau
$$

where $m$ is a positive scalar, and $g$ is another simple function. In this paper, the value of the material integer $m$ is set to 1 . It is important to observe the similarity between this law and the classical laws of damage mechanics [Lemaitre, 1996] which are widely used, for example, to describe the stiffness drop in materials due to microcracking.

\subsubsection{Calculation of $r$}

The diffusion-reaction problem. The calculation of $r$ is carried out through the resolution of a diffusion-reaction problem within the fiber with prescribed boundary conditions. A first analysis is performed based on the following assumptions:

(i) The diffusion-reaction phenomenon is localized near the oxygen input, which means that the effects of the crack's geometry and fiber's size are small; thus, the problem can be written over a semi-infinite unidirectional space.

(ii) The evolution is quasi-stationary, which means that the velocity of the diffusion-reaction process is significantly higher than that of the crack's growth.

(iii) The mechanical and chemical fields are uncoupled, which means, for example, that the reaction and diffusion coefficients are independent of the stress state.

Thus, one must solve the following simple problem (see Figure 2):

- Unknowns (the concentration and flux of $\mathrm{O}_{2}$ within the fiber):

$$
\left\{\begin{array}{l}
c(x, t) \\
\phi(x, t)
\end{array} \quad \forall x \in[a(t) ;+\infty[\quad \forall t \in[0,+\infty]\right.
$$

- Conservation equation:

$$
\frac{\partial \phi}{\partial x}+\phi_{r}=0 \quad \forall x \quad \forall t
$$

- Diffusion equation (Fick's law, $D$ being the diffusion coefficient of $\mathrm{O}_{2}$ in $S i C)$ :

$$
\phi=-D \cdot \frac{\partial c}{\partial x} \quad \forall x \quad \forall t
$$


- Reaction equation (first-order reaction, $k$ being the reaction coefficient of $\mathrm{O}_{2}$ with $\left.\mathrm{SiC}\right)$ :

$$
\phi_{r}=k \cdot c \quad \forall x \quad \forall t
$$

- Boundary condition ( $\phi_{0}$ being a given boundary function):

$$
\phi(x=a(t), t)=\phi_{0}(t) \quad \forall t
$$

- Initial condition:

$$
c(x, t=0)=0 \quad \forall x
$$

- Crack's growth ( $v_{0}$ being a given velocity function):

$$
\dot{a}(t)=v_{0}(t) \quad \forall t
$$

whose solution is:

$$
\left\{\begin{array}{l}
c(x, t)=\frac{\phi_{0}(t)}{\sqrt{k D}} \cdot \exp \left(-\sqrt{\frac{k}{D}}(x-a(t))\right) \\
\phi(x, t)=\phi_{0}(t) \cdot \exp \left(-\sqrt{\frac{k}{D}}(x-a(t))\right)
\end{array}\right.
$$

Calculation of $r$. Thus, with $m=1, r$ is equal to:

$$
r(x, t)=\int_{0}^{t} \frac{k \phi_{0}(t)}{\sqrt{k D}} \cdot \exp \left(-\sqrt{\frac{k}{D}}(x-a(t))\right) d \tau
$$

and the crack's velocity is introduced through a change of variable:

$$
r(x, t)=\int_{a_{0}}^{a(t)} \frac{k \phi_{0}\left(a^{-1}(a)\right)}{\sqrt{k D}} \cdot \exp \left(-\sqrt{\frac{k}{D}}(x-\alpha)\right) \frac{d \alpha}{\dot{a}\left(a^{-1}(a)\right)}
$$

If the diffusion-reaction process zone is small compared to the size of the crack:

$$
\sqrt{\frac{k}{D}}(x-a)\left\{\begin{array}{rrr}
=0 & \text { when } & x=a \\
\gg 1 & \text { when } & x>a
\end{array}\right.
$$

which means that the reaction is essentially a surface reaction, the exponential term of the previous integral simplifies to:

$$
\int_{a_{0}}^{a(t)} \exp \left(-\sqrt{\frac{k}{D}}(x-\alpha)\right) d \alpha \approx\left\{\begin{array}{rrr}
1 / \sqrt{k / D} & \text { when } & x=a \\
0 & \text { when } & x>a
\end{array}\right.
$$

and $r$ simplifies to:

$$
r(x, t) \approx\left\{\begin{array}{rrr}
\frac{k \phi_{0}(t)}{\sqrt{k D}} \frac{1}{\sqrt{k / D}} \frac{1}{\dot{a}(t)} & \text { when } & x=a(t) \\
0 & \text { when } & x>a(t)
\end{array}\right.
$$

Therefore, from now on, we will consider the surface term

$$
r(t)=r(a(t), t)=\frac{\phi_{0}(t)}{\dot{a}(t)}
$$

alone. 


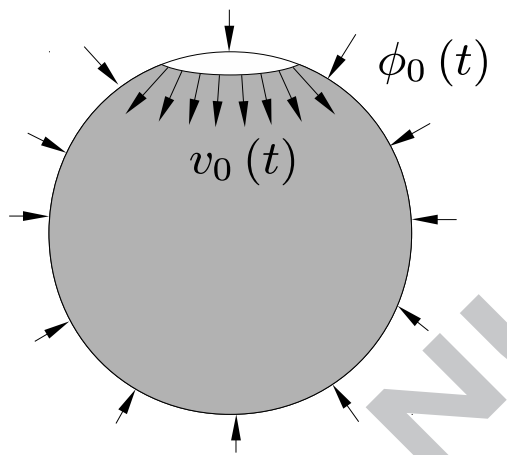

(a) The real problem

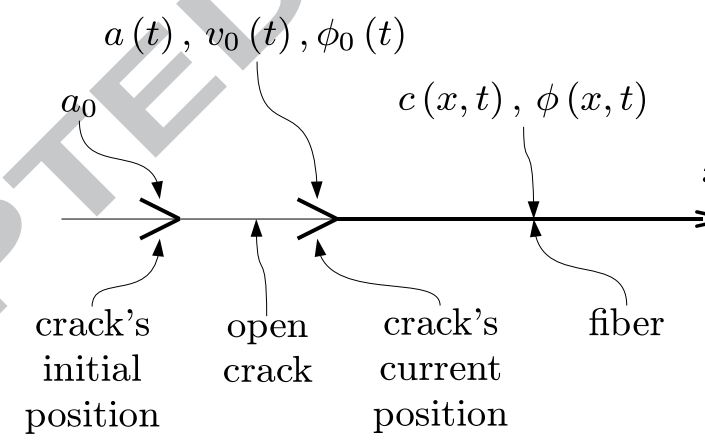

(b) The simplified unidirectional problem

Figure 2: The diffusion-reaction problem for the calculation of $r$ 
Return to the damage law. Finally, the damage law for $m=1$ is:

$$
K_{s c}(t)=K_{c}\left(1-g\left(\frac{\phi_{0}(t)}{\dot{a}(t)}\right)\right)
$$

where $\phi_{0}(t)$ is the incoming oxygen flux at the crack's tip, i.e. the reaction rate of the oxidizing surface reaction, and $\dot{a}(t)$ is the crack's velocity. In the general case, for $m \geq 0$, one has:

$$
K_{s c}(t)=K_{c}\left(1-g\left(\frac{\phi_{0}^{m}(t)}{\dot{a}(t)} \cdot \frac{1}{m} \cdot\left(\frac{k}{D}\right)^{\frac{m-1}{2}}\right)\right)
$$

\subsubsection{Choice of $g$}

There are several possible choices for $g$. Some of these choices are compared in Figure 3:

1. Taking $g(r)=\lambda r^{n}$ (where $\lambda$ and $n$ are positive scalars), Equation (24) reduces to:

$$
\frac{K_{s c}(t)}{K_{c}}=1-\lambda\left(\frac{\phi_{0}(t)}{\dot{a}(t)}\right)^{n}
$$

which has no physical meaning for large values of $r$ (see Figure 3): $K_{s c}<0$.

2. Taking $g(r)=e^{-\lambda / r^{n}}$ (where $\lambda$ and $n$ are positive scalars), Equation (24) reduces to:

$$
\frac{K_{s c}(t)}{K_{c}}=1-\exp \left(-\lambda\left(\frac{\dot{a}(t)}{\phi_{0}(t)}\right)^{n}\right)
$$

which is physically more meaningful (see Figure 3 ).

3. Another possibility is to use the first order of the Taylor series expansion of the previous choice, leading to $g(r)=1-\lambda / r^{n}$. Then, Equation (24) reduces to:

$$
\frac{K_{s c}(t)}{K_{c}}=\lambda\left(\frac{\dot{a}(t)}{\phi_{0}(t)}\right)^{n}
$$

which has no physical meaning for small values of $r$ (see Figure 3): $K_{s c}>K_{c}$. However, in practice, such values would never be reached because the subcritical propagation is replaced by the classical propagation when $K_{s c}$ reaches $K_{c}$. Therefore, this will be our choice from now on.

\subsubsection{Summary}

Finally, each part of the model has a clear physical meaning: 


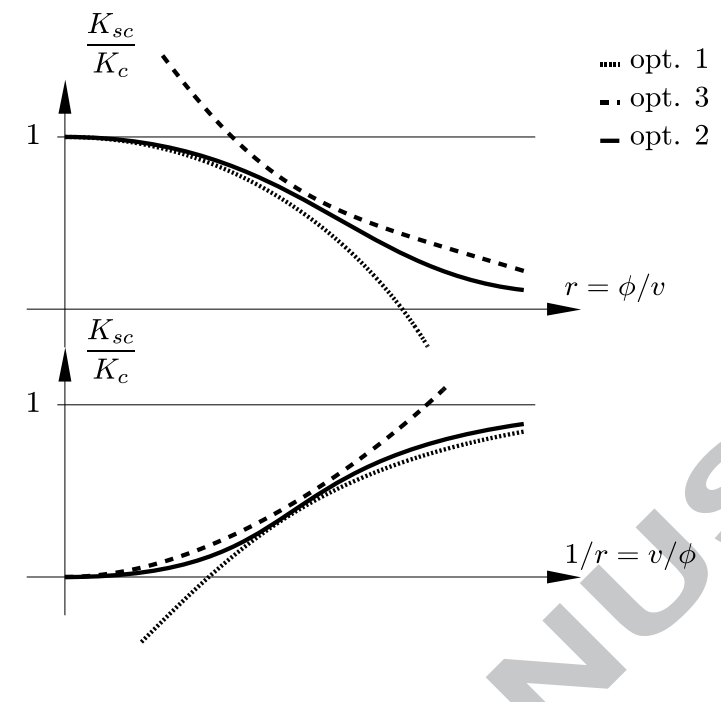

Figure 3: Comparison of several options for $g$

- Equation (6) is the standard equation of fracture mechanics. Since the damaging reaction is a surface reaction (see Equation (23)), the subcritical propagation is stable and continuous. Thus, during the whole propagation $(\dot{a}(t)>0)$, Equation (6) reduces to:

$$
K(a(t), t)=K_{s c}(t)
$$

- The damage laws (27) and (28) also have a clear interpretation: the lower the velocity and/or the higher the reaction rate, the smaller the SCSIF and, conversely, the higher the velocity and/or the lower the reaction rate, the larger the SCSIF. The power law defined by $\lambda$ and $n$ characterizes the degradation of the CSIF for a given amount of oxygen arriving at the crack's tip. This is an intrinsic material law which depends neither on the temperature nor on time.

Grouping Equations (2) for the crack's intensity, (29) for the crack's propagation and (27) or (28) for the material's degradation through oxidation, this theory produces a differential problem in $a(t)$ which describes the crack's subcritical propagation under any mechanical loading and any chemical environment. This can lead to several models depending on the complexity of the description of the chemical environment surrounding the crack.

It is worth mentioning that in the case of Choice (28), if the reaction rate is assumed to be constant over time, one reverts to the framework of classical Paris-based laws (see Equation (5)) by intro- 
ducing Equation (29):

$$
\dot{a}(t)=\frac{\phi_{0}}{\lambda^{1 / n} K_{c}^{1 / n}} \cdot K^{1 / n}
$$

\section{A first model with a simple environment, for low temperature}

\subsection{The model}

\subsubsection{Fiber environment}

In this section, we consider that the fiber's surface is in direct contact with the ambient air. Thus, the reaction rate at the crack's tip is given by:

$$
\phi_{0}=k \cdot c_{0}
$$

where $k$ is the reaction coefficient between $\mathrm{O}_{2}$ and $\mathrm{SiC}$, which is assumed to be independent of mechanical fields such as the stress field or the strain field, and whose temperature dependence is assumed to be of the Arrhenius type ( $k_{0}$ being the prefactor and $E_{a}^{k}$ the activation energy):

$$
k=k_{0} \cdot \exp \left(-\frac{E_{a}^{k}}{R T}\right)
$$

and $c_{0}$ is the $O_{2}$ concentration in the surrounding air, related to the partial $O_{2}$ pressure through the ideal gas law:

$$
c_{0}=\frac{p_{\mathrm{O}_{2}}}{R T}
$$

\subsubsection{Resolution}

The fiber's time-to-rupture, denoted $t_{R}$, is the solution of the differential problem $(m=1)$ :

$$
\left\{\begin{array}{l}
a(t=0)=a_{0}\left(\sigma_{R}\right) \\
\dot{a}(t)=\frac{k c_{0}}{\lambda^{1 / n} K_{c}^{1 / n}} \sigma^{1 / n} Y^{1 / n} \sqrt{a(t)}^{1 / n} \quad \forall t \\
a\left(t=t_{R}\right)=a_{c}(\sigma)
\end{array}\right.
$$

which, in the case of constant mechanical and chemical loading, can be integrated analytically:

$$
t_{R}=\frac{2 n}{2 n-1} \frac{\lambda^{1 / n} K_{c}^{2}}{k c_{0} \sigma^{1 / n} Y^{2}}\left(\sigma^{(2 n-1) / n}-\sigma_{R}^{(2 n-1) / n}\right)
$$

For more complex loading cases, numerical integration may be required. 
\begin{tabular}{|c|}
\hline$K_{c}(M P a \sqrt{\mu m})$ \\
\hline 1250 \\
\hline
\end{tabular}

Table 1: Toughness of Hi-Nicalon fibers

\begin{tabular}{|c|c|c|}
\hline$L_{0}(\mathrm{~mm})$ & $\sigma_{0}(\mathrm{MPa})$ & $m_{\sigma}$ \\
\hline 1 & 3640 & 8.47 \\
\hline
\end{tabular}

Table 2: Weibull coefficients of Hi-Nicalon fibers

\subsection{Calibration}

\subsubsection{Summary of published data}

For Hi-Nicalon fibers, the toughness, previously presented in [Bunsell and Berger, 2000] and [Gauthier and Lamon, 2009], is given in Table 1 and the Weibull coefficients, previously presented in [Gauthier and Lamon, 2009], are given in Table 2.

\subsubsection{Fitting of the experimental data}

At this stage, the parameters which remain to be calibrated are: $k$, which is temperature-dependent (see Equation (32)), and $\lambda$ and $n$, which are assumed to be temperature-independent. Since the literature does not provide much in terms of experimental data, this calibration is not straightforward. For example, regarding Hi-Nicalon fibers, only constant loading and environmental conditions have been investigated [Gauthier and Lamon, 2009]. These data will be used in this paper. Therefore, in Model (34), only the terms $k / \lambda^{1 / n}$ and $n$ can be calibrated at each temperature. Table 3 presents the result of the calibration process, and Figure 4 shows the comparison of the prediction given by the calibrated model with the experimental data.

\begin{tabular}{|c|c|c|}
\hline$k / \lambda^{1 / n}\left(T=500^{\circ} C\right)$ & $k / \lambda^{1 / n}\left(T=800^{\circ} C\right)$ & $n$ \\
\hline $410^{-5}$ & $110^{-1}$ & 0.13 \\
\hline
\end{tabular}

Table 3: The coefficients related to the subcritical propagation

\subsection{Discussion}

Even though this model is satisfactory at low temperatures, Figure 4 reveals a limitation at high temperatures: the model drastically underestimates the fiber's lifetime, especially in the case of long lifetimes. This is a major problem because the model already contains a parameter to adjust the temperature dependence. What this means is that this temperature dependence is more 


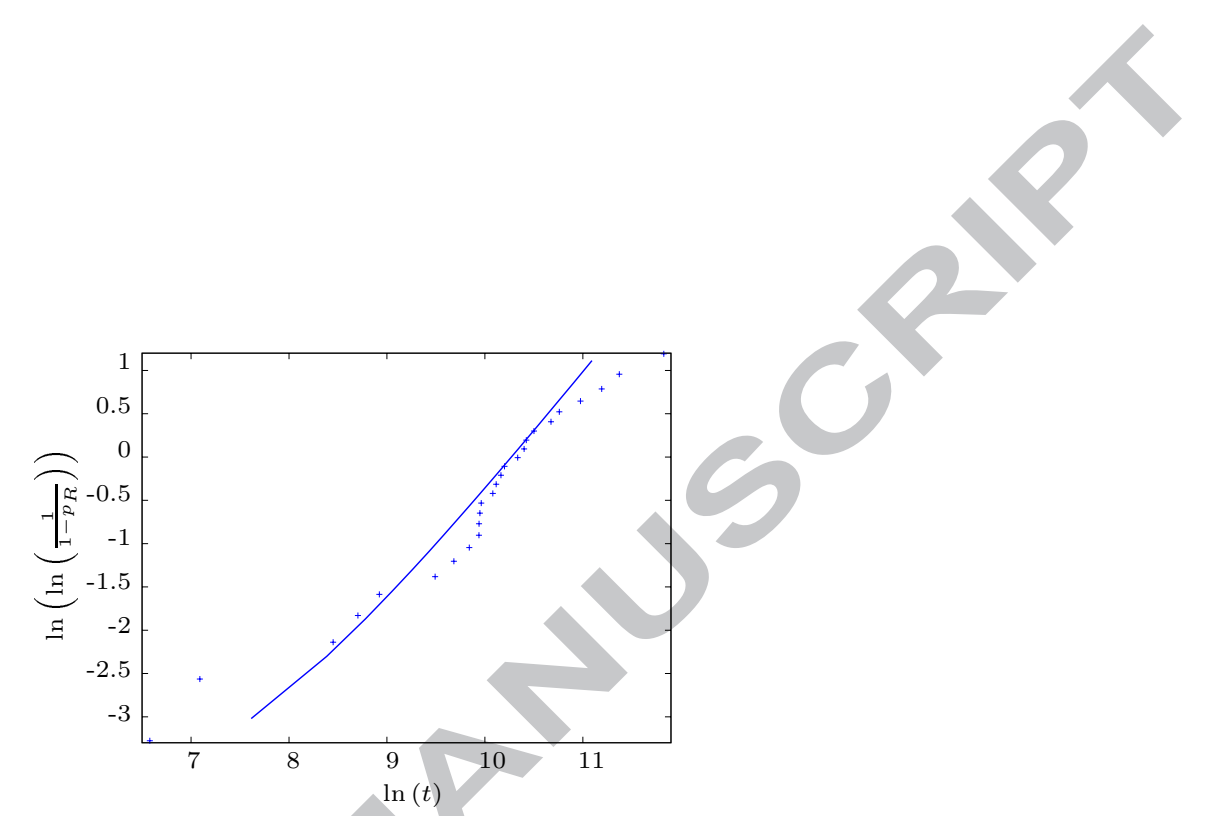

(a) $\mathrm{T}=500^{\circ} \mathrm{C}, \sigma=1500 \mathrm{MPa}$

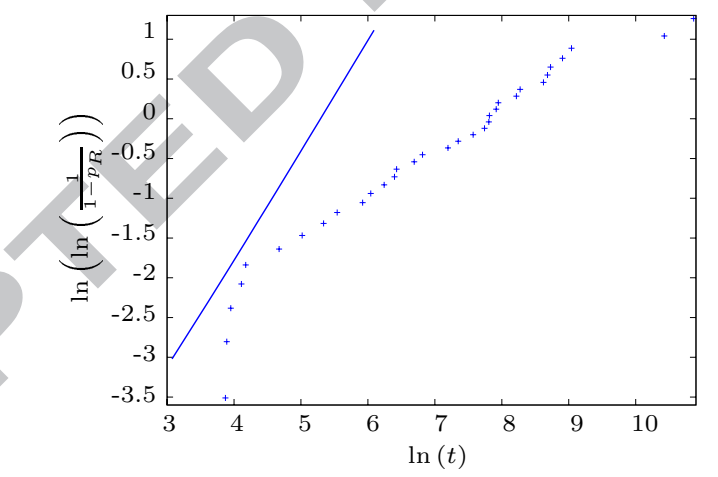

(b) $\mathrm{T}=800^{\circ} \mathrm{C}, \sigma=1100 \mathrm{MPa}$

Figure 4: Cumulative lifetime probability distributions of the fibers: model vs. experiment 
complex than an Arrhenius dependence. Indeed, it is well-known [Lawn, 1974; Wachtman, 1996; Evans et al., 1996] that at high temperatures, after some time, the propagation becomes diffusion-controlled: the oxygen must diffuse through an oxide layer formed at the fiber's surface prior to oxidizing the crack's tip, a process which, by reducing the rate of the reaction which damages the material, results in a considerable increase in the fiber's lifetime. Therefore, it is necessary to resort to a more realistic fiber environment. This will be the subject of the next section of the paper.

One should also observe that in the absence of further information only the coefficients $k / \lambda^{1 / n}$ and $n$ could be calibrated. An illustration of the possible values of $\lambda$ and $k$ is given in Figure 5, where the distance used is a classical norm of the modeling error:

$$
e^{2}=\frac{1}{n^{\exp }} \sum_{i=1}^{n^{\exp }}\left(\ln \left(t_{R}^{\bmod }\right)-\ln \left(t_{R}^{\text {exp }}\right)\right)^{2}
$$

Figure 5: Distance between model and experiment at $T=500^{\circ} \mathrm{C}$ as a function of $\lambda$ and $k$

4. A Second model with a realistic environment, for high -and lowtemperatures

\subsection{The model}

\subsubsection{Fiber environment}

In this section, we use a more realistic model of the oxidizing environment in the vicinity of the fiber. Basically, this is a variation of the framework introduced in [Deal and Grove, 1965], customized for the $\mathrm{SiC}_{(s)}+\mathrm{O}_{2}(\mathrm{~g}) \longrightarrow \mathrm{SiO}_{2}(\mathrm{~s})$ reaction on the surface of the fiber. This enables us to introduce the effect of the oxide layer generated around the fiber which delays the arrival of oxygen at the crack's tip. Assuming that the problem is axisymmetrical and that the thickness 
of the oxide layer is small in relation to the fiber, one can consider a unidirectional model (see Figure 6).

The diffusion-reaction problem. A first analysis is performed with the following assumptions:

(i) The evolution is quasi-stationary, which means that the velocity of the diffusion-reaction process is significantly higher than that of the growth of the oxide layer;

(ii) The mechanical and chemical fields are uncoupled, which means, for example, that the reaction and diffusion coefficients are independent of the stress state.

Thus, one must solve the following simple problem:

- Unknowns (concentration and flux of $\mathrm{O}_{2}$ in $\mathrm{SiO}_{2}$ ):

$$
\left\{\begin{array}{l}
c(x, t) \\
\phi(x, t)
\end{array} \quad \forall x \in[-e(t), 0] \quad \forall t \in[0,+\infty]\right.
$$

- Conservation equation:

$$
\frac{\partial \phi}{\partial x}=0 \quad \forall x \quad \forall t
$$

- Diffusion equation (Fick's law, $D$ being the diffusion coefficient of $\mathrm{O}_{2}$ in $\mathrm{SiO}_{2}$ ):

$$
\phi=-D \cdot \frac{\partial c}{\partial x} \quad \forall x \quad \forall t
$$

- Boundary conditions:

- Reaction condition at $x=-e(t)$ (first-order reaction, $k$ being the reaction coefficient of $\mathrm{O}_{2}$ with $\mathrm{SiC}$ ):

$$
\phi(x=-e(t), t)=k \cdot c(x=-e(t), t) \quad \forall t
$$

- Prescribed concentration at $x=0$ :

$$
c(x=0, t)=c_{0}(t) \quad \forall t
$$

whose solution is:

$$
\left\{\begin{array}{l}
c(x, t)=\left(1+\frac{x}{e(t)}\right) \cdot c_{0}(t)-\left(\frac{x}{e(t)}\right) \cdot \frac{c_{0}(t)}{1+k e(t) / D} \\
\phi(x, t)=\frac{k c_{0}(t)}{1+k e(t) / D}
\end{array}\right.
$$


The oxide layer's growth problem.

- Unknown (the thickness $e$ of the oxide layer):

$$
e(t) \quad \forall t \in[0,+\infty]
$$

- Growth equation (oxide creation, $M$ and $\rho$ being respectively the volume mass and the molar mass of $\mathrm{SiO}_{2}$ ):

$$
\frac{\partial e}{\partial t}(t)=-\frac{\rho}{M} \cdot \phi(x=-e(t), t) \quad \forall t
$$

- Initial condition:

$$
e(t=0)=0
$$

Reaction rate. The reaction rate at the crack's tip is obtained by coupling the previous two problems:

$$
\phi_{0}(t)=\frac{k c_{0}(t)}{1+k e(t) / D}
$$

where the thickness $e$ of the oxide layer is defined by the differential problem

$$
\left\{\begin{array}{l}
\frac{d e}{d t}(t)=\frac{\rho}{M} \cdot \frac{k c_{0}(t)}{1+k e(t) / D} \\
e(t=0)=0
\end{array}\right.
$$

\subsubsection{Resolution}

Finally, the fiber's time-to-rupture, denoted $t_{R}$, is the solution of the differential problem $(m=1)$ :

which can be integrated numerically.

$$
\left\{\begin{array}{l}
a(t=0)=a_{0}\left(\sigma_{R}\right) ; e(t=0)=0 \\
\dot{a}(t)=\frac{1}{\lambda^{1 / n}} \cdot \frac{k c_{0}}{1+k e(t) / D} \sigma^{1 / n} Y^{1 / n} \sqrt{a(t)}^{1 / n} \\
\frac{d e}{d t}(t)=\frac{\rho}{M} \cdot \frac{k c_{0}(t)}{1+k e(t) / D} \\
a\left(t=t_{R}\right)=a_{c}(\sigma)
\end{array}\right.
$$

\subsection{Calibration}

\subsubsection{Summary of published data}

The silica-related coefficients are given in Table 4. The diffusion coefficient of $\mathrm{O}_{2}$ in $\mathrm{SiO}_{2}$, assumed to be Arrhenius-dependent in temperature:

$$
D_{0_{2} / S O_{2}}=D_{0} e^{-\frac{E_{a}^{D}}{R T}}
$$

can be calibrated using the experimental data of [Boyer, 2005] and is given in Table 5 . 


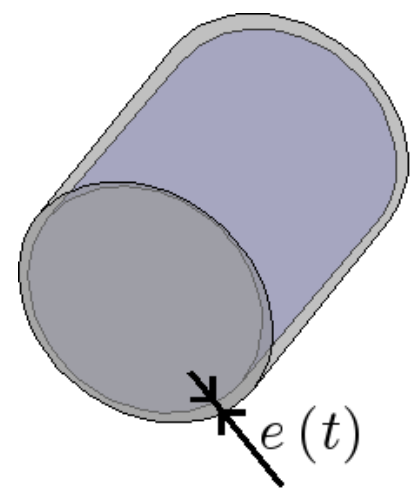

(a) Protective oxide layer

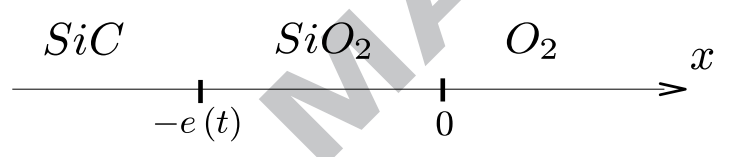

(b) Associated unidirectional problem

Figure 6: The fiber's self-protection problem

\begin{tabular}{|c|c|}
\hline$\rho_{\mathrm{SiO}_{2}}\left(\mathrm{Kg} \cdot \mathrm{mol}^{-1}\right)$ & $M_{\mathrm{SiO}_{2}}\left(\mathrm{Kg} \cdot \mathrm{m}^{-3}\right)$ \\
\hline $60.084310^{-3}$ & 2200 \\
\hline
\end{tabular}

Table 4: Silica properties

\begin{tabular}{|c|c|}
\hline$D_{0}\left(\mathrm{~m}^{2} \cdot \mathrm{s}^{-1}\right)$ & $E_{a}^{D}\left(\mathrm{~J} \cdot \mathrm{mol}^{-1}\right)$ \\
\hline $210^{-8}$ & 177,000 \\
\hline
\end{tabular}

Table 5: Diffusion coefficient of $\mathrm{O}_{2}$ in $\mathrm{SiO}_{2}$ 


\subsubsection{Fitting of the experimental data}

Calibration. In Model (48), $k$ and $\lambda$ remain to be calibrated. Indeed, even though the ratio $k / \lambda^{1 / n}$ was calibrated in the previous section (see Table 3 ), $k$ appears without $\lambda$ somewhere else in the model. Therefore, each parameter must be calibrated separately. In other words, for each temperature, one must find a single pair $(k, \lambda)$ which verifies the relation of Table 3 and makes the model agree with the experimental data. Once again, we used the experimental data of [Gauthier and Lamon, 2009]. The calibrated values are given in Tables 6 and 7 , and Figure 7 shows the comparison of the prediction given by the calibrated model with the experimental data.

\begin{tabular}{|c|c|}
\hline$k\left(T=500^{\circ} \mathrm{C}\right)\left(\mathrm{m} \cdot \mathrm{s}^{-1}\right)$ & $k\left(T=800^{\circ} \mathrm{C}\right)\left(\mathrm{m} \cdot \mathrm{s}^{-1}\right)$ \\
\hline $1.0210^{-10}$ & $2.5610^{-7}$ \\
\hline & \\
\hline$k_{0}\left(\mathrm{~m} \cdot \mathrm{s}^{-1}\right)$ & $E_{a}^{k}\left(\mathrm{~J} \cdot \mathrm{mol}^{-1}\right)$ \\
\hline $1.4710^{2}$ & 180,000 \\
\hline
\end{tabular}

Table 6: Reaction coefficient of $\mathrm{O}_{2}$ with $\mathrm{SiC}$

\begin{tabular}{|c|c|}
\hline$\lambda\left(\mathrm{mol} \cdot \mu \mathrm{m}^{-3}\right)$ & $n$ \\
\hline 0.2 & 0.13 \\
\hline
\end{tabular}

Table 7: Subcritical propagation coefficients

Sensitivity analysis. Figure 8(a) shows the distance between the model and the experiments for the first environmental condition $\left(T=500^{\circ} \mathrm{C}\right)$ near the calibrated values. The large cross represents the calibrated value and the small crosses represent all the values of $(k, \lambda)$ which verify the relation of Table 3. In order to make things clearer, Figure $8(\mathrm{~b})$ shows the distance as a function of the position $\lambda$ along the line. Clearly, the solution is not unique and there are many pairs which minimize the distance. Indeed, at that temperature, the oxide layer is expected to have almost no effect [Boyer, 2005] and, therefore, no information can be retrieved.

Conversely, Figure 9 shows the same plots for the second environmental condition $\left(T=800^{\circ} C\right)$. Clearly, the calibrated pair $(k, \lambda)$ is that which verifies the relation of Table 3 and minimizes the distance between the model and the experiments. This calibration was made possible by the growth of the oxide layer which dissociates $k$ from $\lambda$ in the term $k / \lambda^{1 / n}$.

\subsection{Elements of validation}

Work is currently in progress in order to validate the calibration of this model, but some conclusions can already be drawn. 


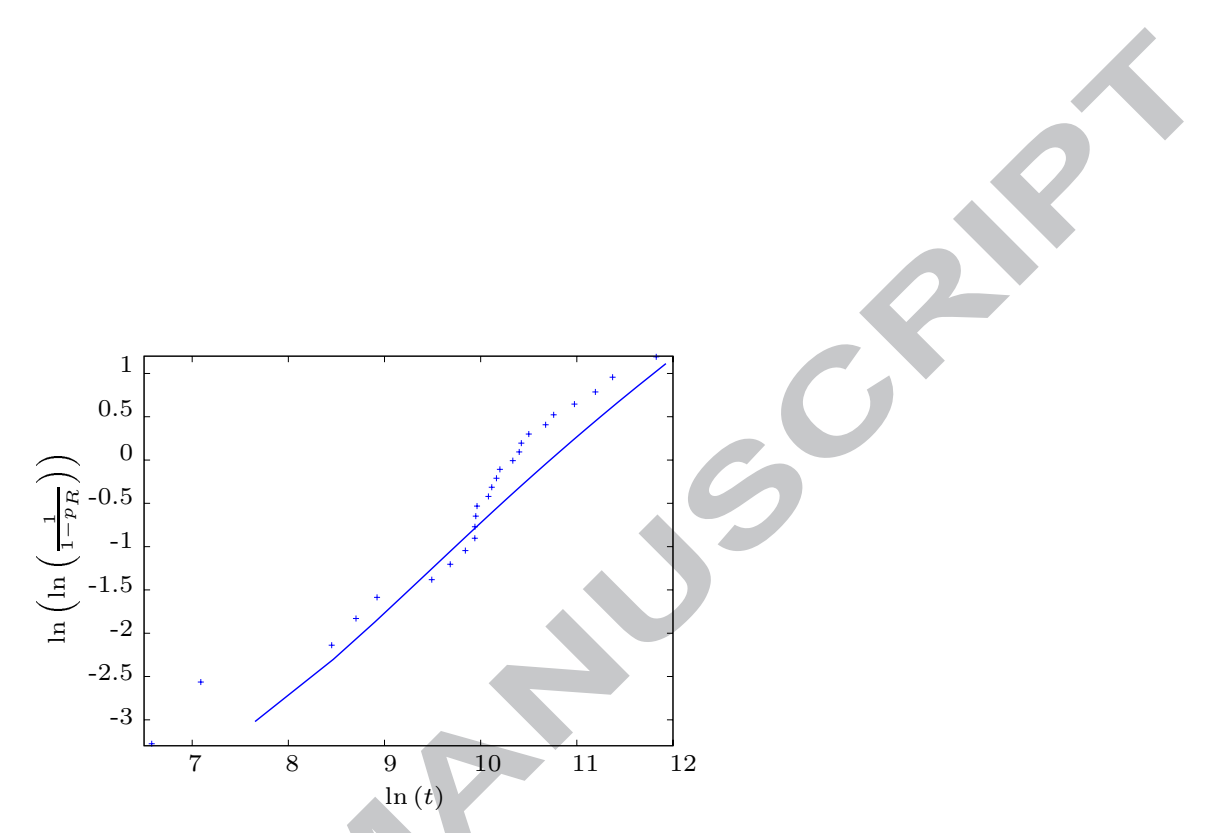

(a) $\mathrm{T}=500^{\circ} \mathrm{C}, \sigma=1500 \mathrm{MPa}$

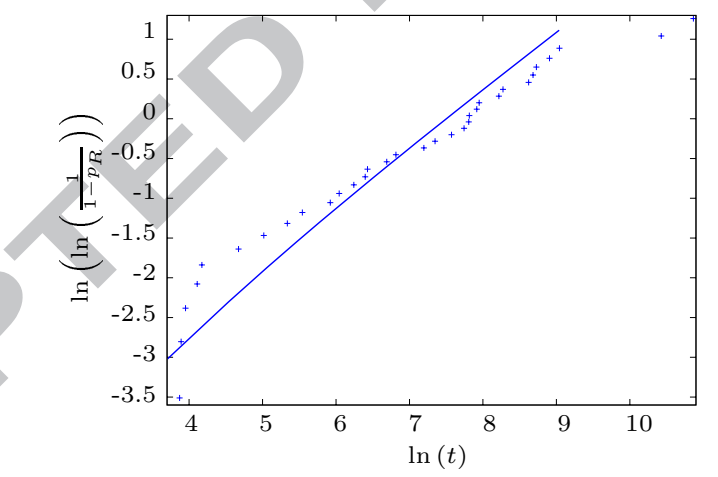

(b) $\mathrm{T}=800^{\circ} \mathrm{C}, \sigma=1100 \mathrm{MPa}$

Figure 7: Cumulative lifetime probability distributions of the fibers: model vs. experiment 


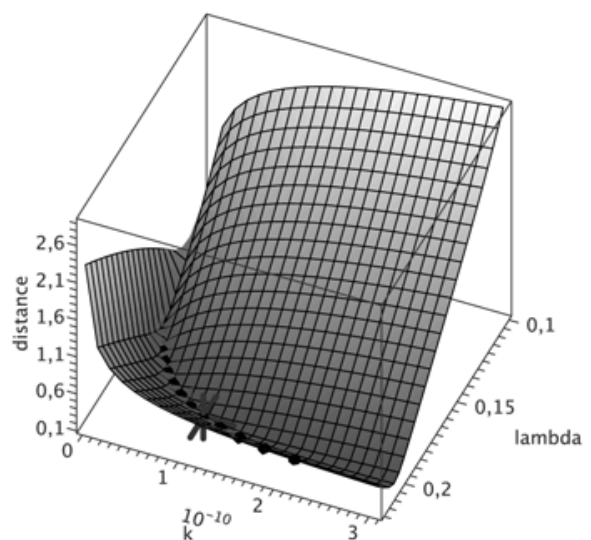

(a) as a function of $\lambda$ and $k$

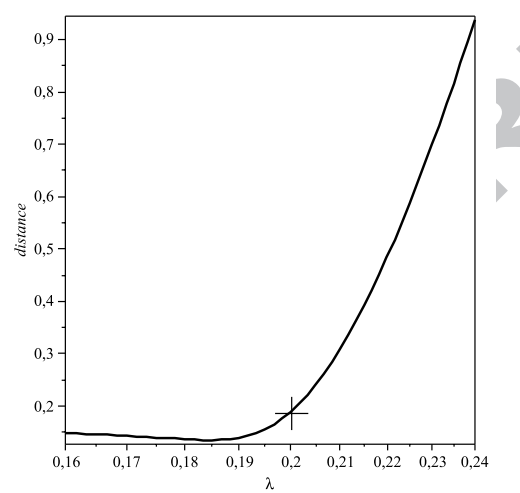

(b) as a function of $\lambda$, with $k / \lambda^{1 / n}$ fixed

Figure 8: Distance between model and experiments at $T=500^{\circ} \mathrm{C}, \sigma=1500 \mathrm{MPa}$

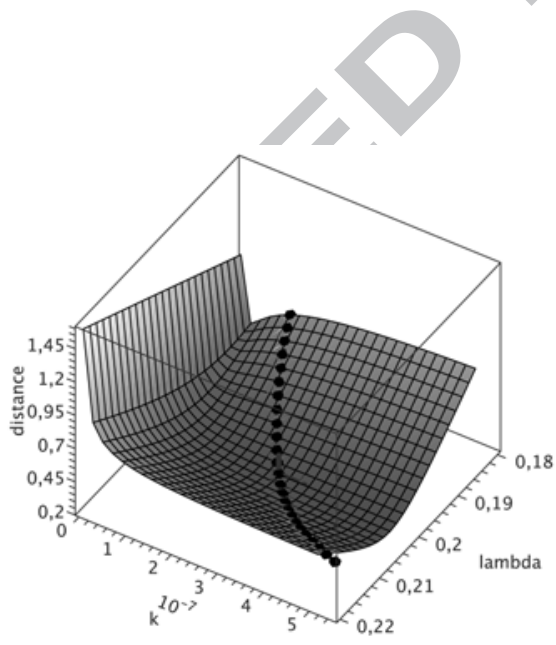

(a) as a function of $\lambda$ and $k$

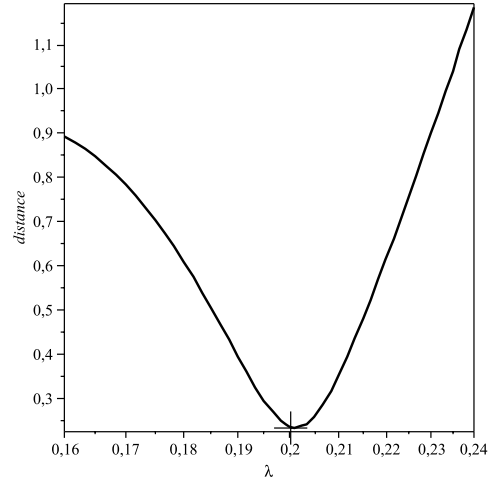

(b) as a function of $\lambda$, with $k / \lambda^{1 / n}$ fixed

Figure 9: Distance between model and experiments at $T=800^{\circ} \mathrm{C}, \sigma=1100 \mathrm{MPa}$ 
Growth of the oxide layer. An element of validation concerning the calibration of the coefficient of the $\mathrm{SiC} / \mathrm{O}_{2}$ reaction (see Table 6) is provided by Figure 10, which compares the predicted (Equation (47)) and experimentally observed (see [Boyer, 2005]) thicknesses of the oxide layer around the fiber as functions of time at several temperatures. As expected [Boyer, 2005], the calibrated reaction coefficient is such that the growth is governed primarily by the diffusion process.

Effect of the partial oxygen pressure. An element of validation concerning the lifetime model directly can also be given. However, not enough data is available on individual Hi-Nicalon fibers and, therefore, one must use data on Hi-Nicalon fiber bundles. Indeed, observing that a fiber bundle consists of a large number of fibers $(\approx 500)$, one can show that its lifetime is subject to only small variations [Phoenix, 1978] and is close to that of a particular fiber, called the critical fiber, characterized by a given probability of failure [Gauthier and Lamon, 2009; Gauthier et al., 2009]. The comparison of the predicted and experimentally observed [Gauthier et al., 2009] fiber bundle lifetimes as functions of the partial oxygen pressure (Figure 11) shows that the orders of magnitude predicted using our model with $m=1$ are correct.

\section{Conclusion}

This paper reexamined the well-established modeling framework for the subcritical propagation of cracks in ceramics. A more general theory for the description of the degradation of the material due to oxidation was introduced and applied to the case of surface defects on ceramic fibers. The analysis showed that the classical Paris law belongs to this framework, and indicated its limits. Two lifetime prediction tools for ceramic fibers were derived from the new framework. These tools differ in the level of complexity with which the fiber's chemical environment is described: in the first tool, the fiber is oxidized directly by the ambient air, whereas in the second tool the oxide layer which surrounds the fibers and delays the oxidation process is taken into account.

As expected, after calibration, the first model was found to reflect reality satisfactorily at low temperatures, at which the oxide layer plays no role, but to be inaccurate at higher temperatures. Conversely, the predictions given by the second model are correct at both low and high temperatures because this model handles reaction-controlled propagation and diffusion-controlled propagation naturally. Moreover, since the framework clearly separates the underlying subcritical propagation mechanisms (the oxidation reaction which affects the local mechanical properties of the fibers and the chemical environment of the fiber which governs the kinetics of the reaction), the calibration can be considered to be valid for any set of environmental conditions (low oxygen pressure, high temperature, variation over time, etc.) provided no other mechanism is involved. Some validation was presented. Work is currently underway in order 


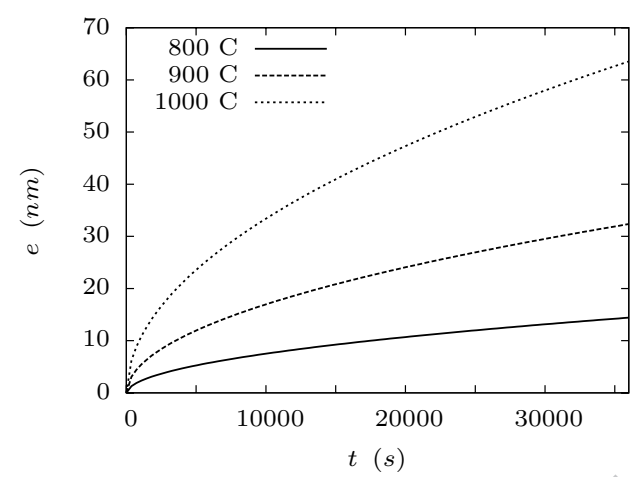

(a) Model

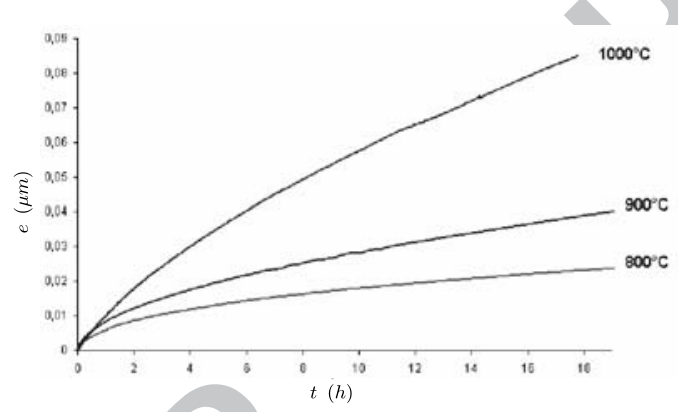

(b) Experiments [Boyer, 2005]

Figure 10: The thickness of the oxide layer as a function of time at various temperatures: model vs. experiments [Boyer, 2005]

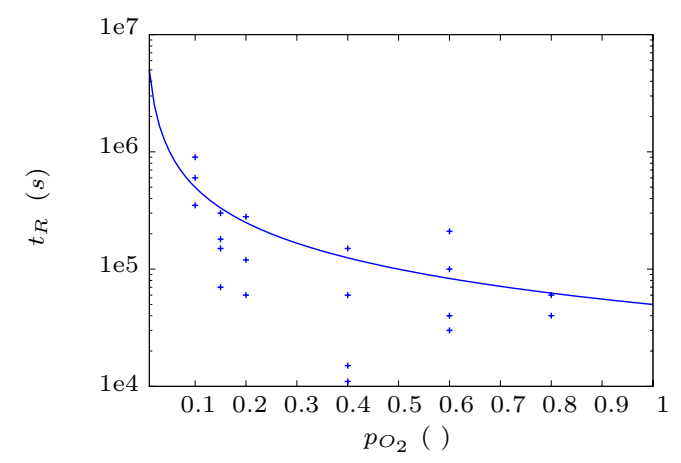

Figure 11: Fiber bundle lifetime vs. partial oxygen pressure, at $T=500^{\circ} \mathrm{C}$ and $\sigma=$ 1,000 MPa: predicted lifetime of the bundle's critical fiber (i.e. the fiber with $5 \%$ failure probability) vs. experiment [Gauthier et al., 2009] 
to extend this validation and define its limits. An interesting application of this approach would be the analysis of accelerated tests (for example using high partial oxygen pressure) in order to reduce the cost of testing campaigns.

Finally, it is important to note that the model is suitable for varying environmental conditions, which constitute the cornerstone of the fiber's lifetime model as a first step toward a complete lifetime model of the tow. For example, in a composite, the oxygen concentration around the fibers may vary due to self-healing of the matrix. Thus, work is underway, with the introduction of self-healing mechanisms, in order to extend this prediction tool from fibers to fiber bundles, then from fiber bundles to complete tows.

\section{References}

Aveston, J., Cooper, G., Kelly, A., 1971. Single and multiple fracture. In: Proc. of the Conference of the National Physical Laboratory on the properties of fiber composites. Vol. 4. p. 15-26.

Boyer, M., 2005. Oxidation/Corrosion of ceramic matrix composites in aircraft type atmospheres (in French). Master thesis, LCTS, Bordeaux I University.

Bunsell, A., Berger, M., 1999. Fine Ceramic Fibers. Marcel Dekker, Inc.

Bunsell, A., Berger, M., 2000. Fine diameter ceramic fibres. Journal of the European Ceramic Society 20 (13), 2249-2260.

Cluzel, C., Baranger, E., Ladevèze, P., Mouret, A., 2008. Mechanical behaviour and lifetime modelling of self-healing ceramic-matrix composites subjected to thermomechanical loading in air. Composites, Part A: Applied Science and Manufacturing.

Dambrine, B., 2006. Which composite materials in turbojets of Snecma Moteurs? (in French). In: Comptes rendus des 14èmes Journées Nationales sur les Composites (JNC14).

Deal, B.E., Grove, A.S., 1965. General relationship for the thermal oxidation of silicon. Journal of Applied Physics 36, 3770-3778.

Evans, A.G., Zok, F.W., McMeeking, R.M., Du, Z.Z., 1996. Models of hightemperature, environmentally assisted embrittlement in ceramic-matrix composites. Journal of the American Ceramic Society 79, 2345-2352.

Evans, A.G., 1997. Design and life prediction issues for high-temperature engineering ceramics and their composites. Acta Materialia 45 (1), 23-40.

Forio, P., Lavaire, F., Lamon, J., 2004. Delayed failure at intermediate temperatures (600 degrees-700 degrees C) in air in silicon carbide multifilament tows. Journal of the American Ceramic Society 87 (5), 888-893. 
Gauthier, W., Lamon, J., 2009. Delayed Failure of Hi-Nicalon and Hi-Nicalon S Multifilament Tows and Single Filaments at Intermediate Temperatures (500 degrees-800 degrees C). Journal of the American Ceramic Society 92 (3), $702-709$.

Gauthier, W., Pailler, F., Lamon, J., and Pailler, R. Oxidation of Silicon Carbide Fibers During Static Fatigue in Air at Intermediate Temperatures. Journal of the American Ceramic Society 92 (9), 2067-2073.

Hild, F., Marquis, D., Kadouch, O., and Lambelin, J.P. Analysis of the failure of ceramics due to subcritical crack growth. Journal of Engineering Materials and Technology 118 (3), 343-348.

Laforêt, A., Lamon, J., 2008. Static fatigue of multifilament tows at high temperatures above $900^{\circ} \mathrm{C}$. In: Proc. of 13th European Conference on Composite Materials (ECCM13).

Lamon, J., 2001. A micromechanics-based approach to the mechanical behavior of brittle-matrix composites. Composites Science and Technology 61, 2259-2272.

Lamon, J., 2007. Brittle fracture and damage mechanics - Probabilisticstatistical Approaches (in French). Hermès - Lavoisier.

Lawn, B., 1974. Diffusion-controlled subcritical crack growth in the presence of a dilute gas environment. Material Science and Engineering 13, 277-283.

Lemaitre, J., 1996. A Course on Damage Mechanics, Second Edition. SpringerVerlag.

Phoenix, S.L., 1978. The asymptotic time to failure of a mechanical system of parallel members. SIAM Journal of applied Mathematics 34 (2), 227-246.

Rebillat, F., Lamon, J., Naslain, R., Lara-Curzio, E., Ferber, M., Theodore, M., 1998. Properties of multilayered interphases in $\mathrm{SiC} / \mathrm{SiC}$ chemical-vaporinfiltrated composites with 'weak' and 'strong' interfaces. Journal of the American Ceramic Society 81, 2315-2326.

Song, D.-Y., Takeda, N., Kawamoto, H., 2000. Corrosion, oxidation and strength properties of Nicalon $\mathrm{SiC}$ fibre under loading. Materials Science and Engineernig 279 (1-2), 82-87.

Wachtman, J. B., 1996. Mechanical properties of ceramics. John Wiley \& Sons, Inc.

Wiederhorn, S., Fuller, E., Thomson, T., 1980. Micromechanics of crack growth in ceramics and glasses in corrosive environments. Metal Science 14, 450-458. 